\title{
Photon strength function deduced from photon scattering and neutron capture
}

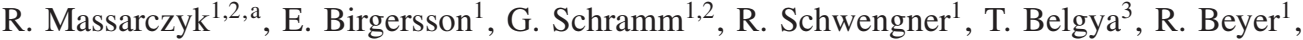 \\ E. Grosse ${ }^{1,2}$, R. Hannaske ${ }^{1,2}$, A.R. Junghans ${ }^{1}$, A. Matic ${ }^{1}$, L. Szentimiklosi ${ }^{3}$, J. Weil ${ }^{3}$, \\ and A. Wagner ${ }^{1}$ \\ 1 Forschungszentrum Dresden-Rossendorf, Germany \\ 2 Technische Universität Dresden, Germany \\ 3 Institute of Isotopes Budapest, Hungary
}

\begin{abstract}
The dipole strength function of ${ }^{78} \mathrm{Se}$ and ${ }^{196} \mathrm{Pt}$ are investigated by two different experimental methods, capture of cold neutrons in ${ }^{77} \mathrm{Se}$ and ${ }^{195} \mathrm{Pt}$ and photon scattering experiments on ${ }^{78} \mathrm{Se}$ and ${ }^{196} \mathrm{Pt}$. Considering the different ways of excitation, the strength function deduced from the results are expected to agree.

The report shows the status of the data analysis and presents first preliminary results.
\end{abstract}

\section{Introduction}

The electric dipole strength of nuclei has a large resonance at excitation energies of about 10-20 MeV, the well known giant dipole resonance (GDR). This distribution can be described by different models. Most common are the descriptions with two [1] or three [2] Lorentzian curves. These have the aim to give an easy description and a wide range parametrization of the photo-nuclear cross section, which is needed for a better understanding in many applications, from providing a database for the construction of the new Generation IV power plants, over the use in network calculations of astrophysical scenarios [3], up to a comparison of theoretical predictions like RPA [4].

Especially in the low energy part of the distribution the strength function does not fit well with the simple Lorentzian explanations. Different nuclei show an enhancement in the strength below the neutron separation energy, for example in ${ }^{90} \mathrm{Zr}$ [5], which is shown in Fig. 1.

There are several approaches to explain this phenomena. The oscillation of a neutron skin against the core, better known as pygmy resonance [7], is frequently mentioned. Other studies proclaim an alternative characterization of the M1 strength [8].

Up to the neutron separation energy experiments using the method of nuclear resonance fluorescence (NRF) have proven their usage as a tool to determine the cross section in this region. At the superconducting electron accelerator ELBE of the research center Dresden-Rossendorf, a bremsstrahlung facility [9] exists, which opens up to study dipole excitations up to the highest neutron separation energies. A short overview will be given in chapter 3. The method of cold neutron capture, shown in chapter 2 , represents an alternative technique to compare the measured data. In order to guarantee equal conditions, two stable nuclei are needed, one with ground state $\mathrm{J}^{P}=\frac{1}{2}^{-}$for neutron capture and one with $\mathrm{J}^{P}=0^{+}$for the scattering experiment. The capture of an s-wave neutron by an nucleus with mass A produces an A +1 excited nuclei with predominantly $1-$ states. This is also the case for the dipole excitation of the $\mathrm{A}+1$ nuclei from ground state $0^{+}$to $\mathrm{J}^{P}=1^{-}$. Not many nuclei in the nuclide chart fulfill these conditions. We used ${ }^{77} \mathrm{Se}$ and ${ }^{78} \mathrm{Se}$ as well as ${ }^{195} \mathrm{Pt}$ and ${ }^{196} \mathrm{Pt}$ for this twin experiment.

a e-mail: r.massarczyk@fzd.de

This is an Open Access article distributed under the terms of the Creative Commons Attribution-Noncommercial License 3.0, which permits unrestricted use, distribution, and reproduction in any noncommercial medium, provided the original work is properly cited. 


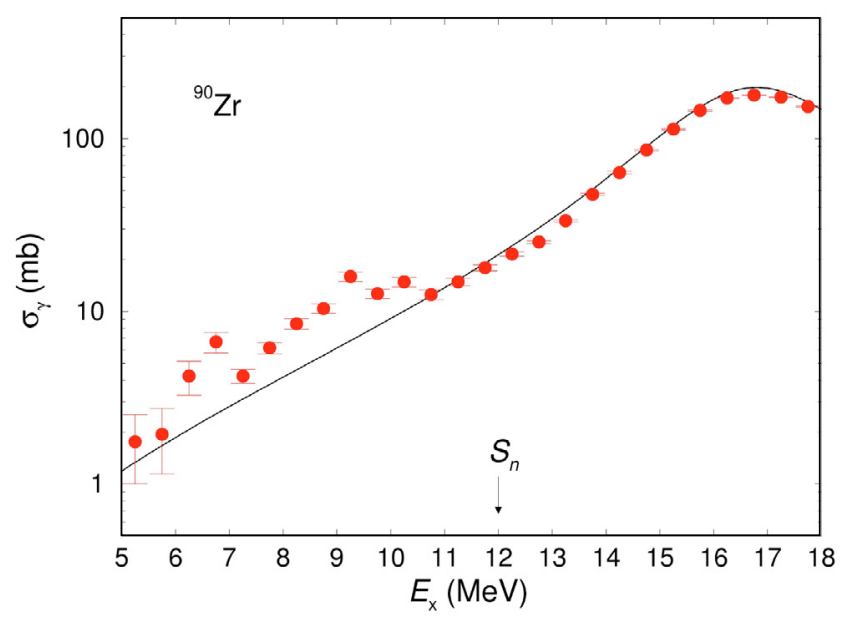

Fig. 1. Total photo-absorption cross section of ${ }^{90} \mathrm{Zr}$ (red dots) and the Lorentz curve with parameters from [6].

After the measurement different simulations have to be performed to understand background events and detector response. For this purpose the GEANT4 [10] package was used.

\section{$2(n, \gamma)$ at IKI Budapest}

\subsection{Experimental side}

The neutron capture experiment took place at the Budapest research reactor, which provides a neutron flux in the thermal energy range of about $2.2 \cdot 10^{14}$ neutrons $/\left(\mathrm{cm}^{2} \cdot \mathrm{s}\right)$ [11] with $10 \mathrm{MW}$ thermal power. The possibility of using the cold neutron source (CNS) allows measurements at the target position with a thermal-equivalent neutron flux of $5 \cdot 10^{7}$ neutrons $/\left(\mathrm{cm}^{2} \cdot \mathrm{s}\right)$. The ${ }^{77}$ Se target was enriched up to $99.66 \%$. After the capture of a cold neutron the nuclei stay in an excited state, which decays via one or more steps back to the ground state. The theoretical aspects of this capture will be discussed in sect. 2.2. These transition are detected with a high-purity germanium (HPGe) detector, which was brought from FZD to Budapest to ensure equal conditions in photon detection. The detector is surrounded by a bismuth germanate (BGO) scintillation detector used as an escape suppression shield. The detector was placed perpendicular to the beam-line at the position of the PGAA (prompt gamma activation analysis) [12]. After subtraction of natural and neutron induced background the measured spectrum was corrected for detector response, which was simulated with GEANT4. The results are shown in Fig. 2 for a nitrogen capture measurement under same conditions. Most of the Compton continuum and the large escape peaks are reduced to a negligible amount. After the correction the cleaned spectra consists of only full energy peaks.

The same algorithm for correcting the spectra was used for the neutron capture of ${ }^{77} \mathrm{Se}$. In Fig. 3 one can see, a broad continuum containing many unresolved peaks. This cleaned spectra is now input for a statistical analysis to deduce the strength function.

\subsection{Statistical analysis}

A statistical analysis has to be done, because not every excited nucleus decays directly to the ground state. For example other calculations in ${ }^{100} \mathrm{Mo}$ [13] show especially in energy ranges up to $8 \mathrm{MeV}$ that the probability for a ground state transition goes on average down to $20 \%$. This implies that most of the deexcitations produce a cascade of photons. A new code has been written, which simulates the deexcitation of the nucleus after the capture, cf. Fig 4. The code follows in principle the idea 


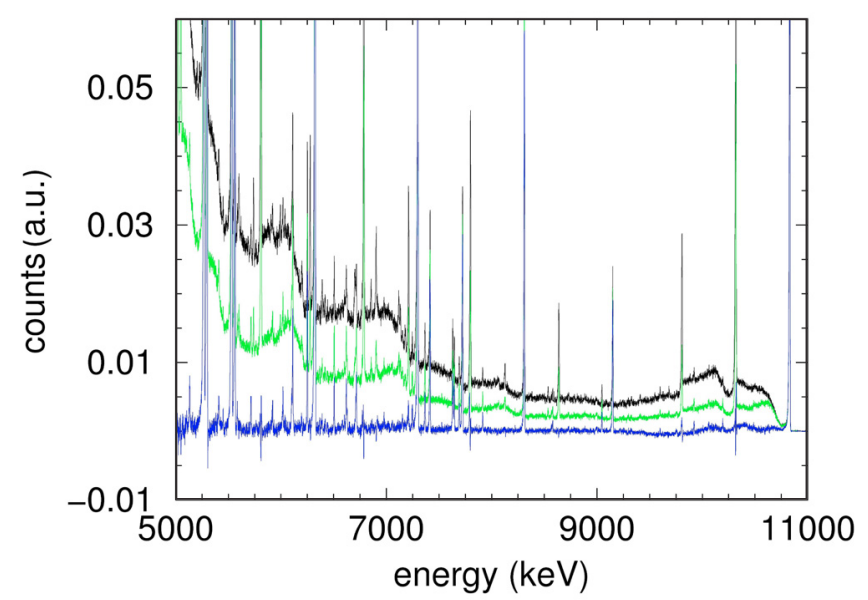

Fig. 2. Spectra of neutron capture in nitrogen, without BGO-Veto (black), with BGO-Veto (green), after correction (blue).

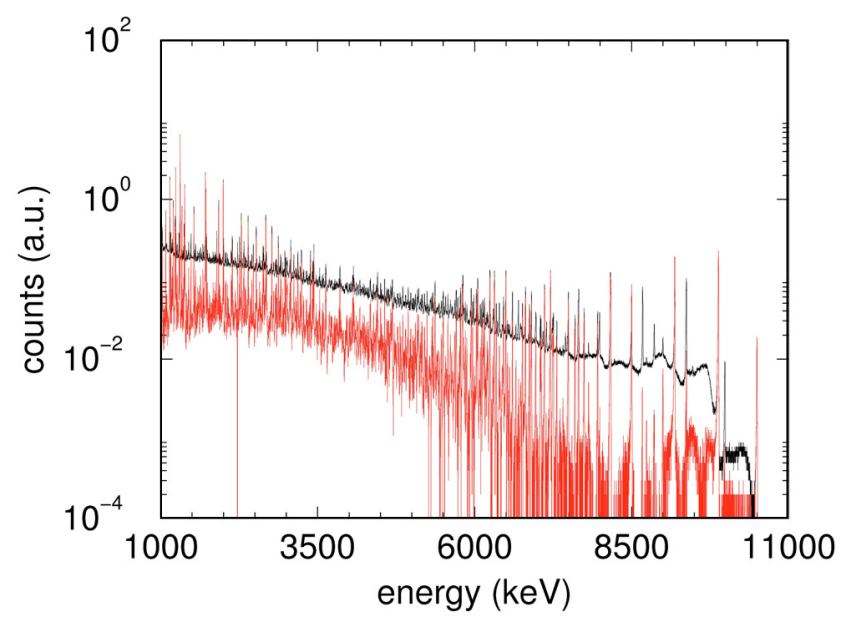

Fig. 3. Spectra of neutron capture in ${ }^{77} \mathrm{Se}$ with BGO-Veto (black) and corrected for detector response (red).

from F. Bečvár [14]. In contrast to this we use a statistical approach, calculating in energy bins of $10 \mathrm{keV}$. This is justified under the assumption, that level density and strength function change smoothly between these energy steps. The advantage is a faster calculation by using the following assumptions:

- the neutrons are captured as s-wave neutrons. This implies a mixture of the start level with spin 0 and 1

- usage of E1, M1 and E2 transitions

- a parametrization of the E1 - GDR with three Lorentzian, cf. Fig. 5 , which fits well the known $(\gamma, \mathrm{n})$ measurements [15]

- parametrization for M1 and E2 according to RIPL-2

- use of discrete levels up to $2.5 \mathrm{MeV}$, beyond this a level density calculated with the constant temperature model [16].

It is possible to reproduce the experimental data with this formalism. In Fig. 6a one can see that the shape of the continuum looks like the experimental one. It is visible in Fig. 6b, that the experimental intensity ratio of the discrete gamma lines is reproduced well by the simulation. For this calculation we did not change the strength function mentioned before. It is work in progress at this point to vary 


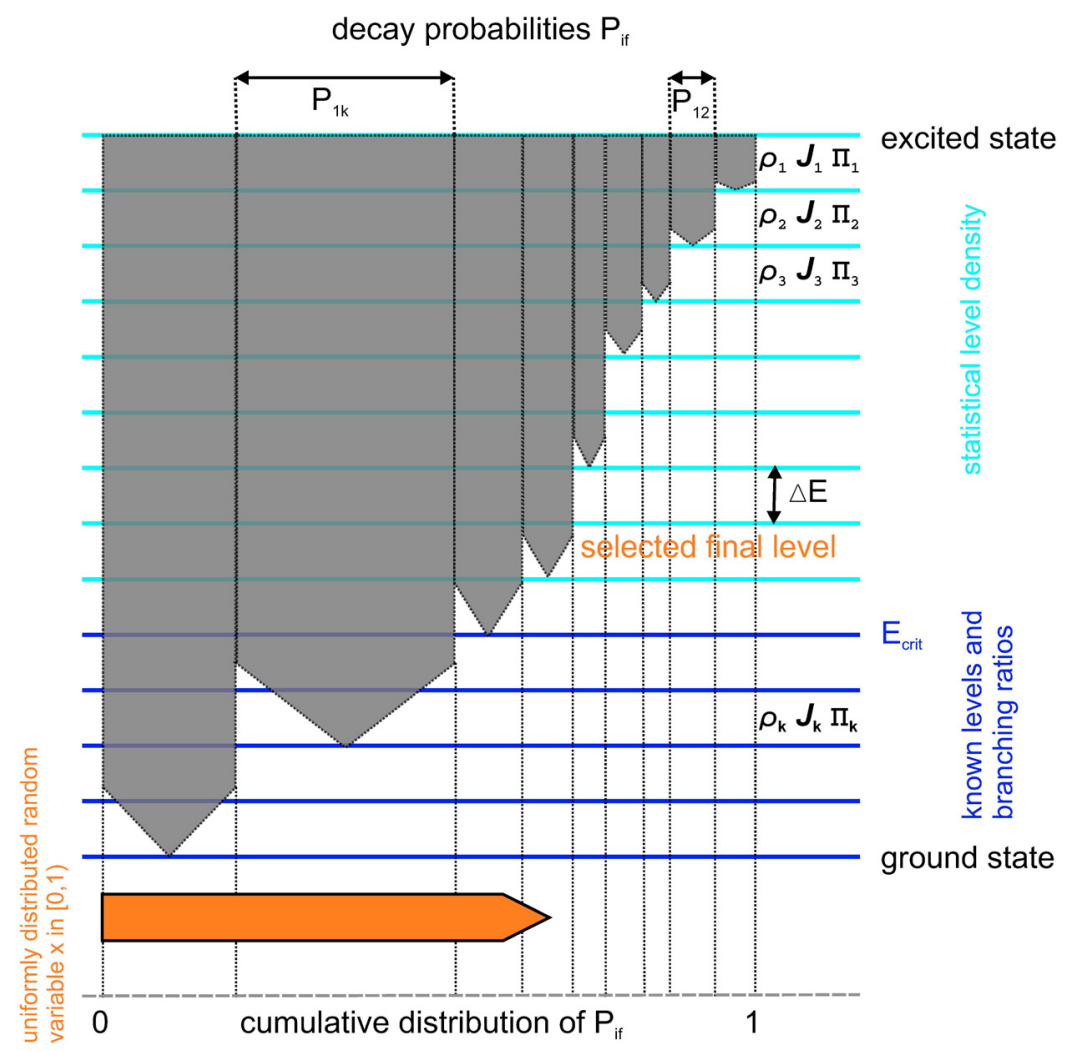

Fig. 4. Working scheme of the code for the decay of one excited state.

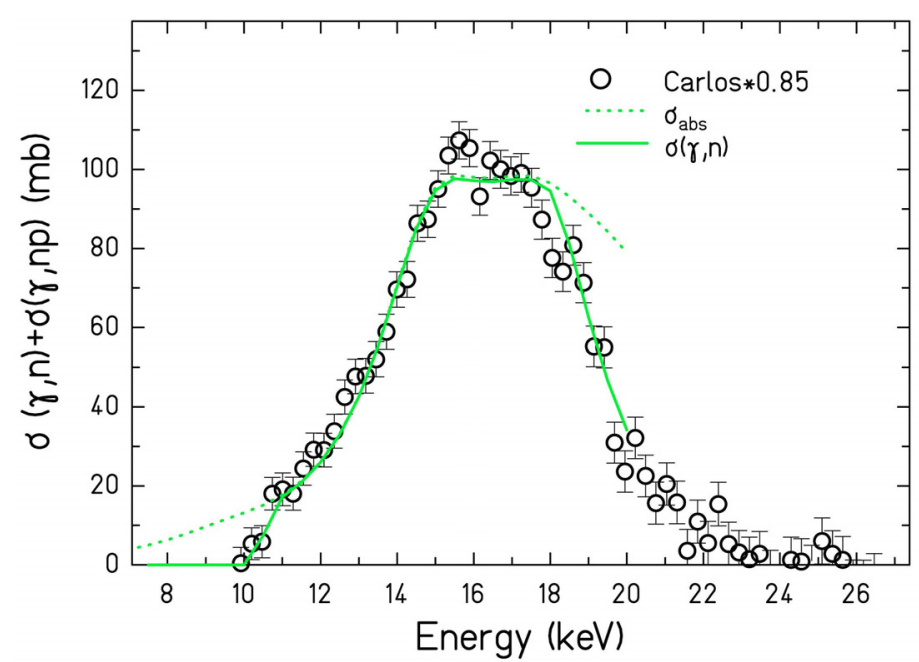

Fig. 5. Comparison of $(\gamma, n)$ data rescaled by 0.85 suggested by Bernan et al., and calculation in TALYS, using parametrization from [2] with parameters $\beta=0.271$ (Raman) and $\gamma=27.1^{\circ}$ (Andrejtscheff). 

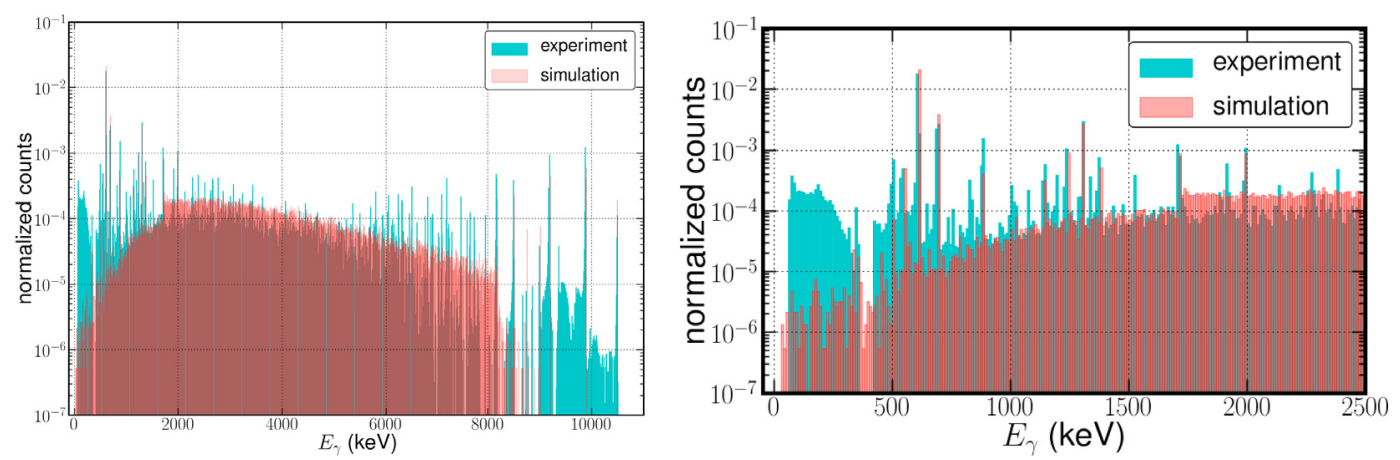

Fig. 6. Results for $\gamma$-cascade simulation (red) and comparison with experimental data (blue).

the strength function in order to reproduce experimental data in the best way. This strength function should be used later to correct the experimental data from Dresden for branching and feeding, cf. end of chapter 3 .

\section{$3(\gamma, \gamma)$ at ELBE in Dresden}

The second part of this double experiment experiment was carried out at the bremsstrahlung facility of the research center Dresden-Rossendorf. The ELBE - accelerator (superconducting Electron Linac with high Brilliance and low Emittance) produces bremsstrahlung at a $7 \mu \mathrm{m}$ thick niobium foil with a maximum electron energy of $18 \mathrm{MeV}$. For the ${ }^{78} \mathrm{Se}\left(\gamma, \gamma^{\prime}\right)$ reaction a kinetic electron energy of 11.5 MeV was used, which guarantees the excitation of states up to the neutron separation threshold of ${ }^{78} \mathrm{Se} \mathrm{S}_{n}=10.49 \mathrm{MeV}$. The collimated photon beam impinged onto the target in a spot of $38 \mathrm{~mm}$ in diameter. The target was a disk of $2 \mathrm{~g}^{78} \mathrm{Se}$ enriched to $99.39 \%$ with a diameter of about $20 \mathrm{~mm}$ to enable an irradiation with a constant flux density over the target area. In front of the target a disk ${ }^{11} \mathrm{~B}$ with a diameter of $20 \mathrm{~mm}$ was placed, with a mass of $0.318 \mathrm{~g}$ and enriched up to $99.5 \%$. Scattered photons were measured with four high-purity germanium (HPGe) detectors of $100 \%$ efficiency relative to a $\mathrm{NaI}$ detector of 3 in. in diameter and 3 in. in length. All HPGe detectors were surrounded by escape-suppression shields made of bismuth germanate scintillation detectors. Two HPGe detectors were placed vertically at $90^{\circ}$ relative to the photon-beam direction at a distance of $28 \mathrm{~cm}$ from the target. The other two HPGe detectors were positioned in a horizontal plane at $127^{\circ}$ to the beam at a

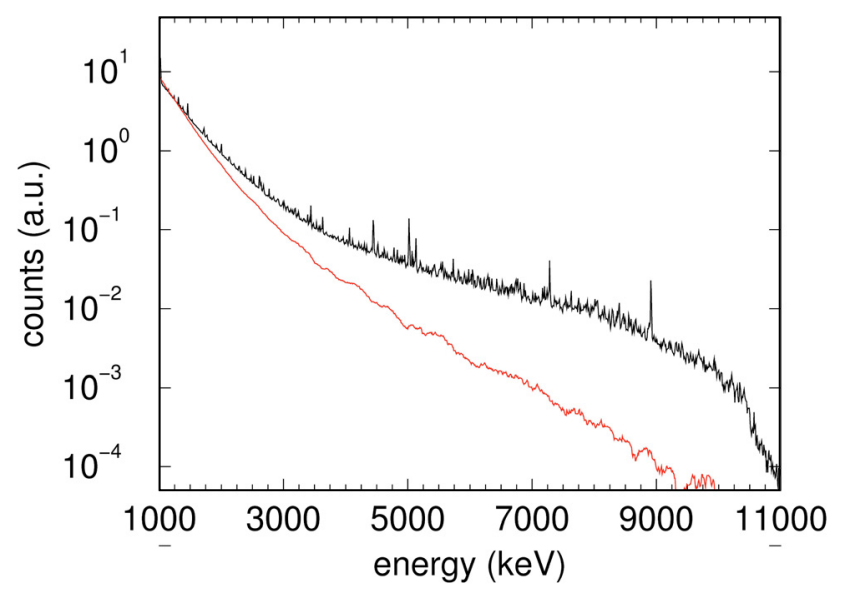

Fig. 7. GEANT4-simulation of the atomic background (red) and experimental measured spectrum (black) for the two detectors under $127^{\circ}$. 


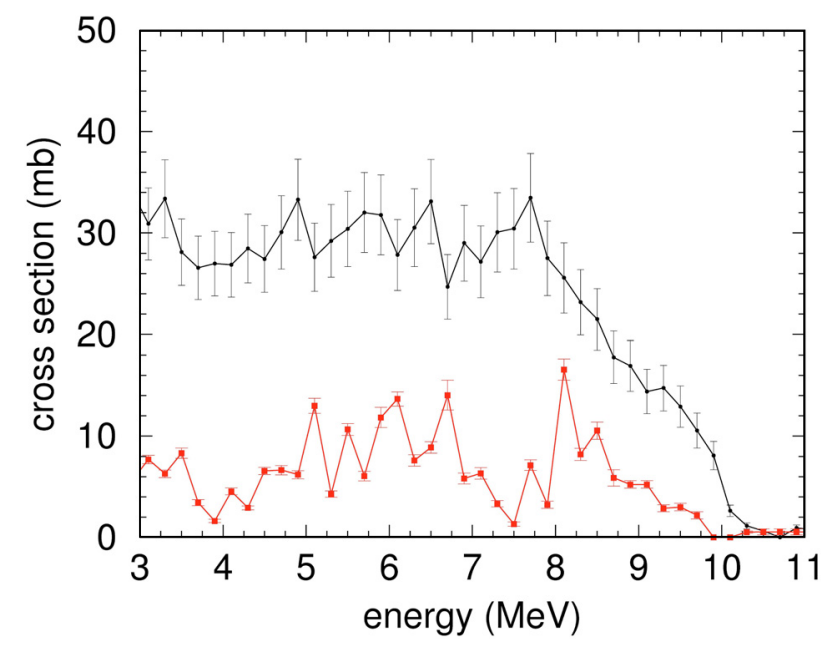

Fig. 8. Cross section from peaks (red) and from peaks and continuum (black) after correction for detector response and atomic background.

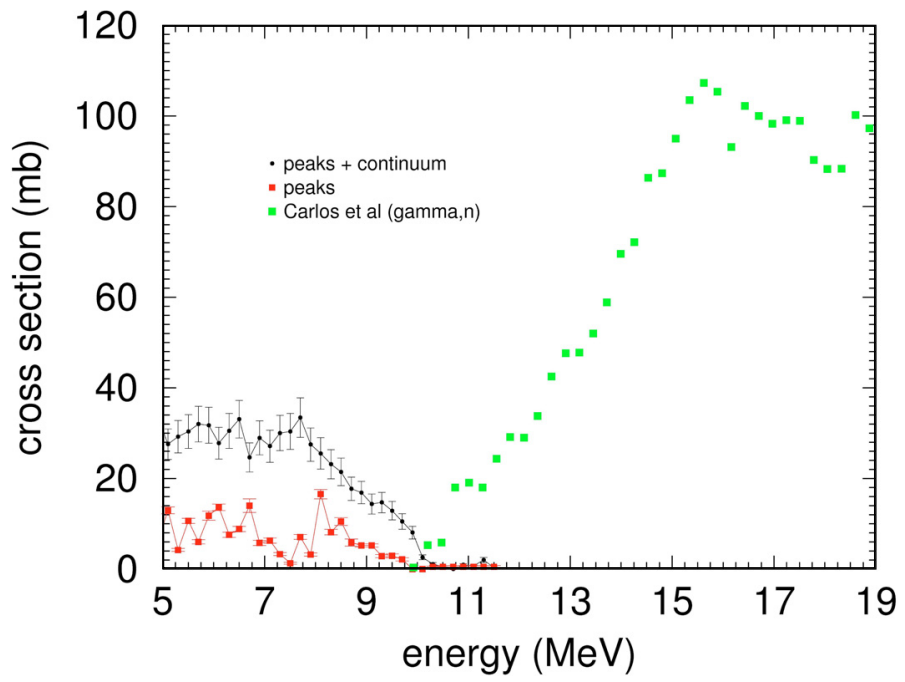

Fig. 9. Relation between uncorrected preliminary data (red and black) and the measurement over the neutron separation threshold (green).

distance of $32 \mathrm{~cm}$ from the target. Absorbers of $8 \mathrm{~mm} \mathrm{~Pb}$ plus $3 \mathrm{~mm} \mathrm{Cu}$ and of $3 \mathrm{~mm} \mathrm{~Pb}$ plus $3 \mathrm{~mm} \mathrm{Cu}$ were placed in front of the detectors at $90^{\circ}$ and $127^{\circ}$.

In photon-scattering experiments the energy-integrated scattering cross section $I_{s}$ of an excited state at the energy $E_{x}$ can be deduced from the measured intensity of the respective transition to the ground state (elastic scattering). It can be determined relative to the known integrated scattering cross sections $I_{s}\left(E_{x}^{\mathrm{B}}\right)$ of states in ${ }^{11} \mathrm{~B}[17]$ :

$$
\frac{I_{s}\left(E_{x}\right)}{I_{s}\left(E_{x}^{\mathrm{B}}\right)}=\left(\frac{I_{\gamma}\left(E_{\gamma}, \theta\right)}{W\left(E_{\gamma}, \theta\right) \Phi_{\gamma}\left(E_{x}\right) N_{N}}\right)\left(\frac{I_{\gamma}\left(E_{\gamma}^{\mathrm{B}}, \theta\right)}{W\left(E_{\gamma}^{\mathrm{B}}, \theta\right) \Phi_{\gamma}\left(E_{x}^{\mathrm{B}}\right) N_{N}^{\mathrm{B}}}\right)^{-1} .
$$

Here, $I_{\gamma}\left(E_{\gamma}, \theta\right)$ and $I_{\gamma}\left(E_{\gamma}^{\mathrm{B}}, \theta\right)$ denote the measured intensities of a considered ground-state transition at $E_{\gamma}$ and of a ground-state transition in ${ }^{11} \mathrm{~B}$ at $E_{\gamma}^{\mathrm{B}}$, respectively, observed at an angle $\theta$ to the beam. $W\left(E_{\gamma}, \theta\right)$ and $W\left(E_{\gamma}^{\mathrm{B}}, \theta\right)$ describe the angular correlations of these transitions. The quantities $N_{N}$ and $N_{N}^{\mathrm{B}}$ 
are the numbers of nuclei in the ${ }^{78} \mathrm{Sr}$ and ${ }^{11} \mathrm{~B}$ targets, respectively. The quantities $\Phi_{\gamma}\left(E_{x}\right)$ and $\Phi_{\gamma}\left(E_{x}^{\mathrm{B}}\right)$ stand for the photon fluxes at the energy of the considered level and at the energy of a level in ${ }^{11} \mathrm{~B}$, respectively.

To determine this cross section the measured spectra had to be corrected for detector response, which was analogue to the one we used for Budapest, and for non-nuclear scattered events. The atomic background was also simulated with GEANT4, where it is possible to switch off nuclear processes. A large left over continuum which remains after subtracting is visible in Fig. 7. About 76 percent of the cross section are in the continuum of unresolvable peaks. Figure 8 shows the cross section from peak and continuum (black) and the cross section just determined by the peaks (red).

At least a correction for branching and feeding is needed. A considered level can be fed by transitions from higher-lying states. The measured intensity of the ground-state transition is in this case higher than the one resulting from a direct excitation only. As a consequence, the integrated cross section deduced from this intensity contains a part originating from feeding in addition to the true integrated scattering cross section. Furthermore, a considered level can deexcite not only to the ground state, but also to low-lying excited states (inelastic scattering). In this case, not all observed $\gamma$ transitions are ground-state transitions. The formalism is the same like in part 2.2. The only difference is that there is now not a single entry level. In photon scattering each level can be excited. The correction has to be done for the whole spectrum. This work is in progress now. As a preliminary result Fig. 9 shows the uncorrected cross section, which is in the same order of magnitude like the data from Carlos et al. [15]. We expect a good continuity to this data after the correction.

\section{References}

1. S.S. Dietrich and B.L. Berman, Atomic Data and Nuclear Data Tables 38, 199 (1988)

2. A.R. Junghans et al., Phys let. B 670, 200 (2008)

3. K. Langanke and M. Wiescher, Rep.Prog. Phys 64, 1657 (2001)

4. W. Kleinig et al., Phys. Rev C 78, 044313 (2008)

5. R. Schwengner et al., Phys. Rev. C 78, 064314 (2008)

6. B.L. Berman et al., Phys. Rev. 162, 1098 (1967)

7. U. Kneissl et al., J. Phys. G: Nucl. Part. Phys. 32 R217-R252 (2006)

8. K. Heyde et al., arXiv:1004.3429v2, (2010)

9. R. Schwengner et al., Nucl. Instrum. Methods A 555, 211 (2005)

10. S. Agostinelli et al., Nucl. Instrum. Methods A 506, 250 (2003)

11. http://www.efnudat.eu/docs/iki.pdf

12. Zs. Révay et al., Nucl. Instrum. Methods B 213, 385 (2004)

13. G. Rusev et al., Phys. Rev. C 77, 064321 (2008)

14. F. Bečvár, Nucl. Instrum. Methods A 417, 434 (1998)

15. P. Carlos et al., Nuclear Physics, Section A Vol. 258, p. 365 (1976)

16. T. von Egidy and D. Bucurescu, Phys. Rev. C 80, 054310 (2009)

17. F. Ajzenberg-Selove and J.H. Kelley, Nucl. Phys. A506, 1 (1990) 